\title{
Histopathological changes in venous grafts and in varicose and non-varicose veins
}

\author{
A K Charles, G A Gresham
}

\begin{abstract}
Aims-To examine veins histologically from different sites in the body to study the effect of venous pressure; and to examine veins used as aortocoronary grafts.

Methods-The axillary vein, femoral vein at the inguinal ligament, the short saphenous vein at the knee and the long saphenous vein at the ankle were removed from 24 necropsy cases of patients aged 2 months to 80 years. Fifteen varicose saphenous veins and 12 aortocoronary grafts removed at surgery were obtained. All were examined histologically.

Results-Varying degrees of intimal thickening composed of collagen, elastin, and smooth muscle were found. These changes were most noticeable in the varicose veins. Intimal changes were also seen related to valves and to adjacent arteries. No clinically relevant lipid was seen in the native veins, though atheromatous changes were seen in the grafts.

Conclusions-Venous changes are related to venous pressure, to local haemodynamic effects, and probably to hypoxia. The changes are often focal and seem to be sequential in their formation. True atheroma is seen in the aortocoronary grafts but is not seen in native veins and this may be the result of additional factors.
\end{abstract}

Intimal changes of varying degrees appear in veins. This is particularly the case in grafts used to bypass diseased coronary arteries. Intimal thickenings also appear in other veins and particularly in varicose veins in the legs. The intravenous pressure at the ankle can be quite high in patients with varicose veins because of incompetent perforating veins.

\section{Methods}

The veins examined were the axillary veins, femoral veins at the inguinal ligament, short saphenous veins at the knee and long saphenous veins at the ankle. These were obtained from 24 necropsy cases of varying ages (from 2 months to 80 years). Varicose veins from 15 surgical cases were also obtained. Twelve saphenous vein aortocoronary bypass grafts were removed at repeat surgery.

Multiple transverse sections were taken from all the vessels. They were stained with haematoxylin and eosin, Weigert's resorcin fuchsin for elastic tissue, and Ponceau $S$ for collagen; alcian Blue at $\mathrm{pH} 2.5$ was used to demonstrate the presence of mucopolysaccharides. Frozen sections were stained with Oil Red $\mathrm{O}$ to show lipids. Paraffin wax sections were also stained with Oil Red O mounted in Apathy's medium: this shows oxidised lipids which are insoluble in the usual dehydrating agents. All the vessels had been fixed in buffered formol-saline.

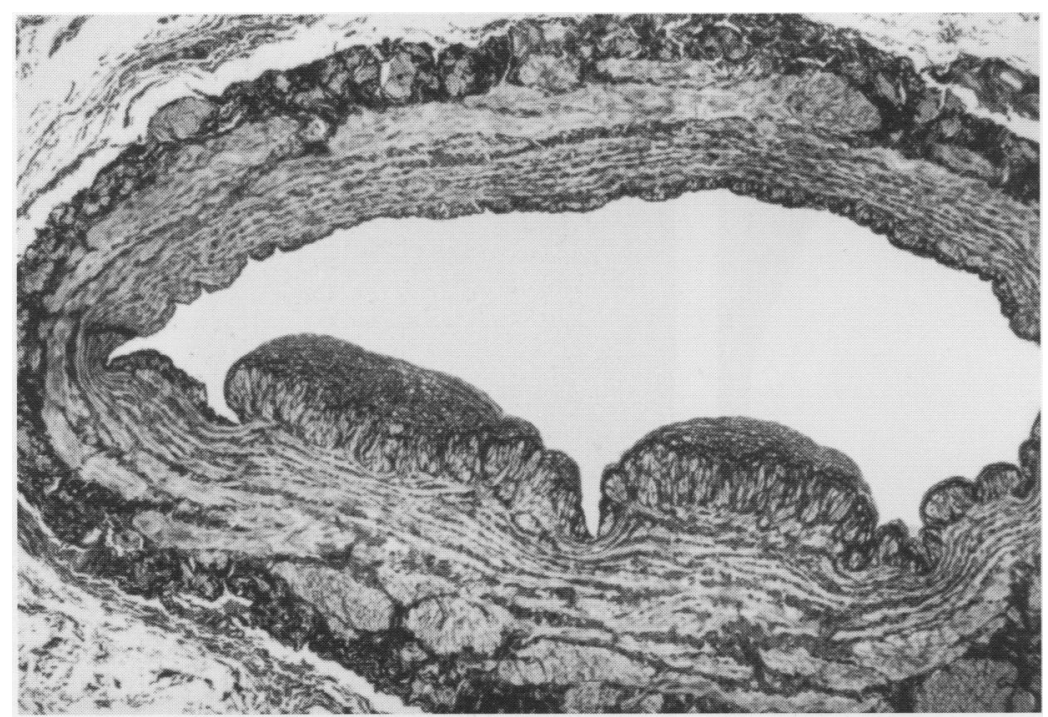

Figure 1 Transverse section of vein showing focal intimal thickenings consisting of collagen, elastin, and muscle with duplicated internal elastic lamina and muscular hypertrophy (Weigert's resorcin fuchsin and Ponceau $S$ (EPS)).
Results

NORMAL VEINS FROM DIFFERENT SITES

The object was to compare appearances in supported veins, such as the axillary and femoral, with unsupported subcutaneous veins, such as the long and short saphenous.

The axillary veins showed the most mild intimal changes and these occurred in the older patients. Intimal changes occurred more frequently in the femoral veins and were seen as early as 20 years of age. Femoral venous intimal thickenings did not always affect the whole circumference of the vessel. They were sometimes, but not always, found in the vein on the side of the distortion caused by an adjacent artery in the pattern, as described by Lev et al. ${ }^{1}$ Sections of the long and short saphenous veins showed more elaborate intimal thickenings with abundant smooth muscle, especially in the saphenous vein at the ankle. In many cases it was curious how focal the lesions were, and they did not always affect the full circumference of the vein (fig 1).

The intimal changes consisted of a layer of 
Figure 2 Intimal thickening (I) showing several layers of deposition (EPS).

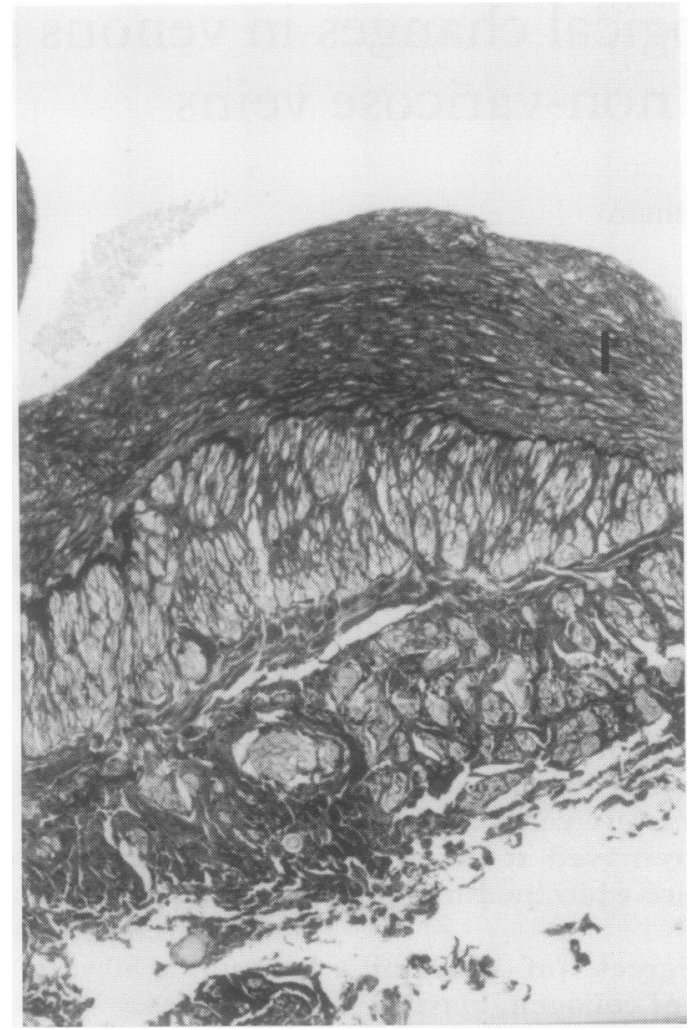

collagen and elastin of variable thickness. The internal elastic lamina was duplicated. In more severe examples the thickening seemed to be laminated, suggesting episodic deposition of fibres (fig 2). Variable amounts of mucopolysaccharides were found in the thickened intima and in the adjacent media. No lipid was seen.

Smooth muscle cells were often seen in the thickened intima, interspaced between the duplicated internal elastic lamina. They seemed to be migrating into the intima from the media passing between and splitting the internal elastic lamina. A new layer of muscle cells running longitudinally was also sometimes found external to the internal elastic lamina, interspaced between it and the medial muscle.

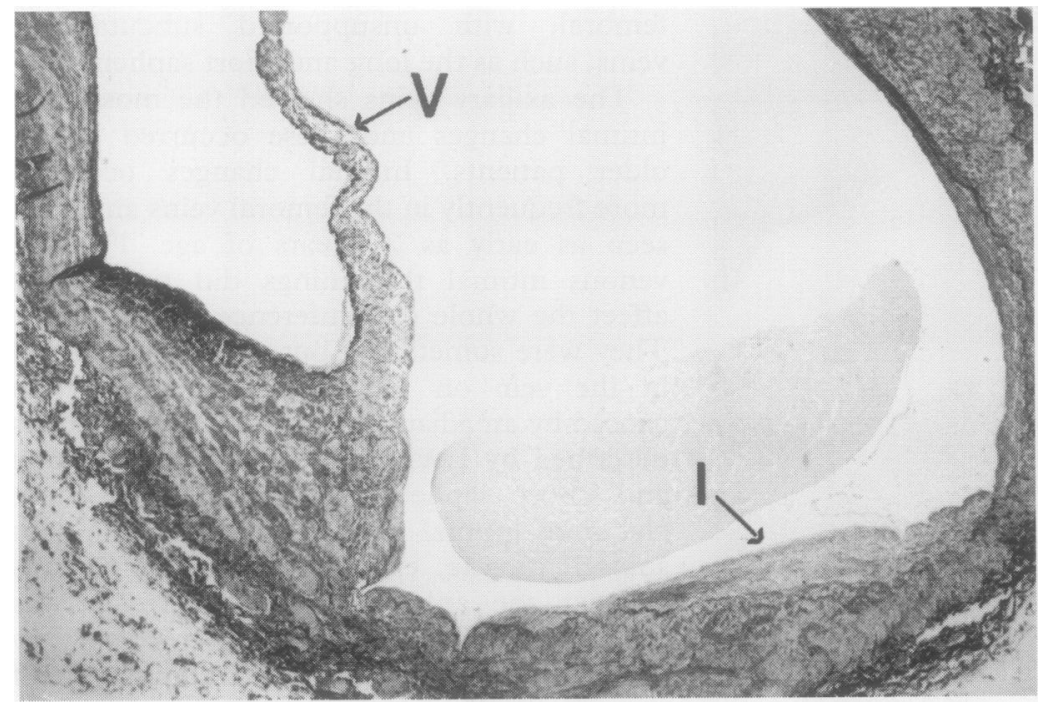

Figure 3 Valve pocket showing the intimal changes in the valve pocket. $I=$ intimal change, $V=$ Valve leaflet (EPS).
The adventitia and media displayed muscular hypertrophy and an increase in elastic tissue.

\section{VARICOSE VEINS}

Most of these were surgically removed specimens, a few were taken at necropsy. Macroscopically some specimens had greatly thickened walls, others were thin and collapsed.

The histological appearances were essentially similar to those seen in the normal saphenous veins but of a greater degree. The neointima was well developed in most of the samples. Hyperplasia of longitudinally arranged smooth muscle was also observed in the media and adventitia.

Mucopolysaccharides occurred in both intima and media and foci of hyaline fibrous tissue were sometimes seen in relation to such deposits. Calcification was occassionally found. Lipid deposition was not seen.

The degree of fibroelastic thickening overlying the muscular neointima varied considerably. It was notably different on opposing sides of venous valves often occurring behind the valve cusps, suggesting the importance of haemodynamic factors in their genesis (fig 3). Occasional organising thrombi were found in the lee of the valve cusps, suggesting that this may be a factor in the development of the intimal lesions (fig 4).

\section{SAPHENOUS GRAFTS}

The saphenous grafts had been in position for varying periods and were removed because of failure to function. They showed intimal thickening consisting of muscular and fibroelastic thickening finally culminating in lipid

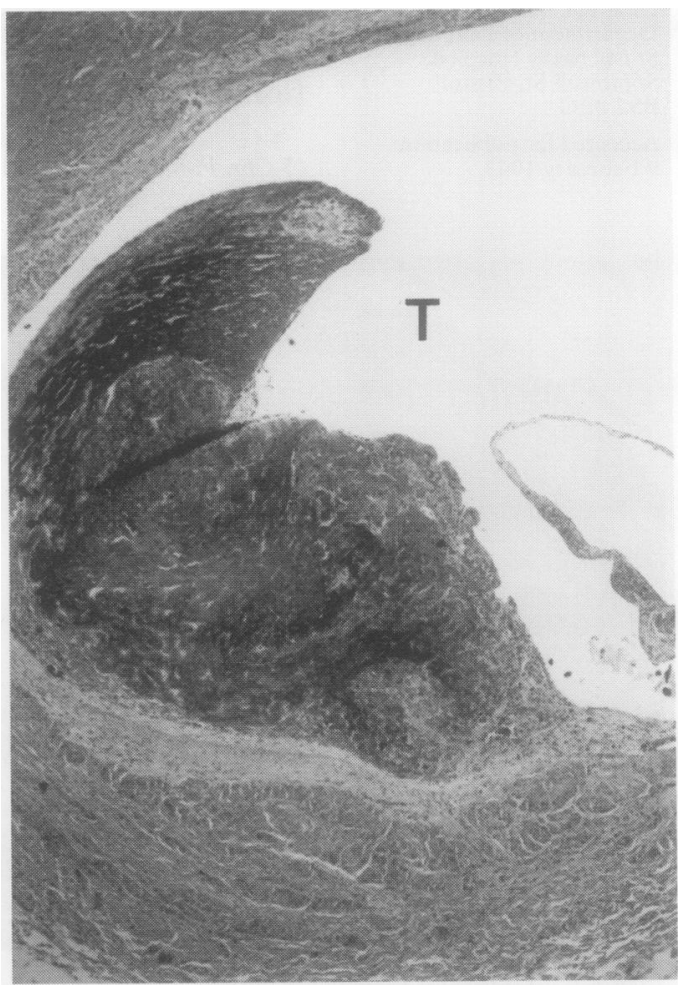

Figure 4 Valve pocket with organising thrombus. $T=$ thrombus (EPS). 


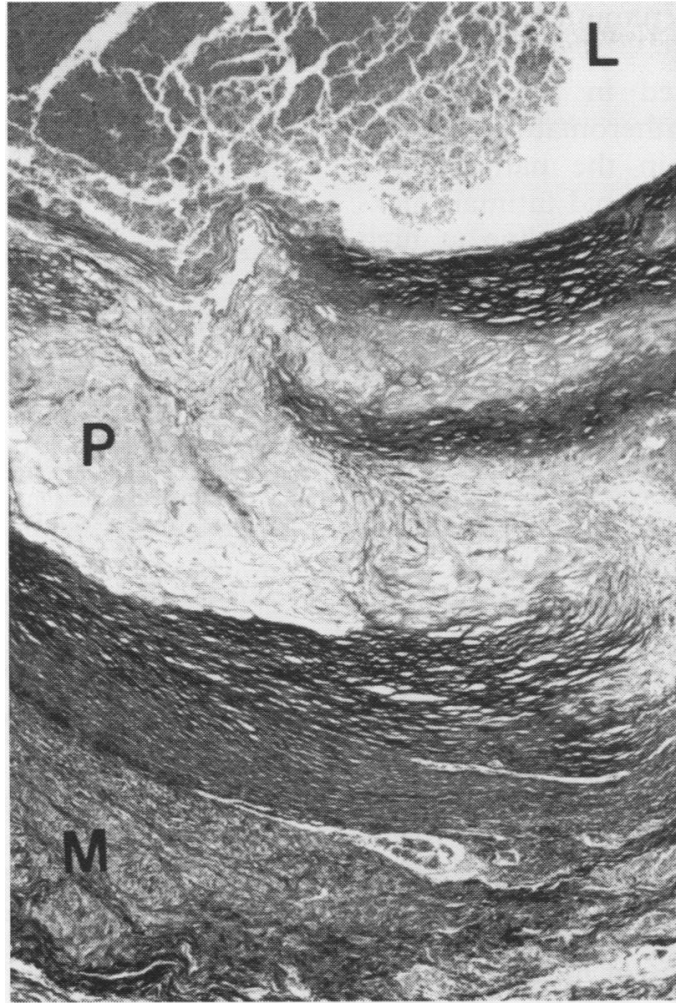

Figure 5 Saphenous aortocoronary with an atheromatous plaque including extensive lipid. $L=$ lumen, $P=$ plaque, $M=$ media (EPS).

deposits (fig 5). These changes have been well described before. ${ }^{2-5}$ We did not see any acute changes due to the endothelial damage caused by the preparation of these grafts.

The changes resemble the neointimal changes seen in the varicose and saphenous veins but many have also developed atheromatous changes with lipid.

\section{Discussion}

Considerable differences in intimal thickening were observed in the axillary, femoral, and saphenous veins at necropsy. The neointima was best developed in the most dependent and unsupported vessels such as the long saphenous vein. The differences in the veins taken at necropsy can be partially attributed to the intraluminal pressures at different sites. Similar differences have previously been observed in portal, splenic, and mesenteric veins and in the inferior vena cava and have been attributed to pressure differences in the vessels. ${ }^{16}$ Stuart et al found lipid in the plaques seen in mesenteric veins in portal hypertension. ${ }^{6}$ We did not find lipid in any of our veins.

Changes in varicose veins were more severe than in normal veins in the same locations. Increased intraluminal pressure due to incompetent perforating veins with little support from the surrounding tissues is likely to be an important factor in these changes, but the possibility of hypoxia due to venous stagnation cannot be excluded. In the areas adjacent to the valves local haemodynamic factors and possibly thrombosis are probably also important. Varicose veins are irregularly

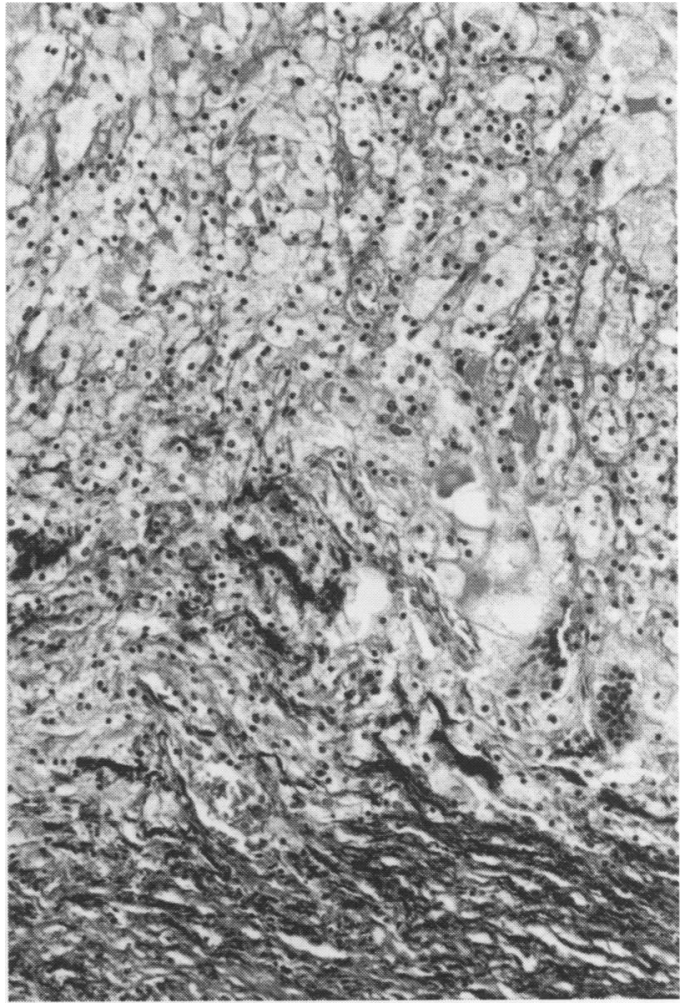

Figure 6 Aortocoronary graft showing foamy macrophages (EPS).

dilated and it is reasonable to postulate the existence of turbulent flow in some of the varices. The presence of laminated fibroelastic intimal thickenings supports this view. Possibly this and other factors may produce intimal thickenings by platelet deposition, thrombosis, and thrombus incorporation in the intima. The presence of laminated fibroelastic intimal thickenings suggests an episodic formation of the thickenings. The different degrees of intimal thickening on either side of venous valves suggests that the valves cause local haemodynamic disturbances. There may be pressure differences across the valves, stagnation of flow behind the cusps, or turbulence or boundary separation in blood flow past the valves. These may help induce thrombosis and hence directly or indirectly lead to intimal thickening.

The muscle in the intima, media, and the adventitia may reflect longitudinal stretching of the vein. Similar longitudinal deposition of muscle has been reported in longitudinally stretchable prosthetic grafts, reflecting the lines of stress. ${ }^{7}$

Intimal smooth muscle cells probably arise as a result of migration from the media and seem to be actively involved in collagen synthesis. This is probably a response to the venous hypertension, but it is also the production of platelet derived growth factors such as platelet derived growth factor (PDGF) and fibroblastic growth factor (FGF) which seem to be chemotactic and mitogenic to fibroblasts and smooth muscle cells. These factors and similar are produced by endothelium, macrophages, and smooth muscle cells as well as platelets. The endothelium can be induced to produce PDGF by 
interleukin 1 (IL1) and tumour necrosis factor. (TNF). ${ }^{89}$

Saphenous veins used in aortocoronary bypass grafts showed atheromatous lesions. These were not seen in the native veins. Saphenous veins develop rapid intimal thickening if prior perfusion with saline at high pressure $(500 \mathrm{~mm} \mathrm{Hg})$ has been done. ${ }^{1011}$ Perfusion at lower pressure $(100 \mathrm{~mm} \mathrm{Hg})$ and using anticoagulated blood resulted in the development of considerably less intimal thickening and longer survival of the grafts. ${ }^{1011}$ High pressure perfusion damages the endothelium causing the release of cytokines, with the development of platelet aggregation, thrombus formation, and fibroblastic and smooth muscle proliferation. ${ }^{4}$ Lipid accumulation often occurs and seems to be related to the degree of hyperlipidaemia in the subject. Veins used as grafts are also effectively devascularised. The loss of the vasa vasorum supply and the intimal thickening reducing oxygenisation by luminal perfusion means the vessel walls are hypoxic. ${ }^{3412}$

This study of veins from various sources indicates the importance of pressure and blood flow in the genesis of intimal thickening and venous changes, and that additional factors are implicated in the development of atheromatous lesions in venous arterial grafts.

1 Lev M, Saphir O. Endophlebohypertrophy and phlebosclerosis. Pictures of Pathology 1951;51:154-78.

2 Barboriak J, Pintar K, Van Horn DL, Batayias GE, Korns ME. Pathologic findings in the aortocoronary Korns ME. Pathologic findings in the

3 Kern WH, Wells WJ, Meyer BW. The pathology of surgically excised aortocoronary saphenous vein bypass grafts. Am $\mathcal{F}$ Surg Pathol 1981;5:491-6.

4 Cox JL, Chiasson DA, Gotlieb AI. Stranger in a strange land: The pathogenesis of saphenous vein graft stenosis with emphasis on structural and functional differences between veins and arteries. Prog Cardiovasc Dis 1991;34:45-68.

5 Sottiurai VS, Yao JST, Flinn WR, Batson RC. Intimal hyperplasia and neointima; an ultrastructural analysis of thrombosed grafts in humans. Surgery 1983;93:809-17.

6 Stuart M, Magery FR. The great veins in venous hypertension. ₹ Pathol Bacteriol 1960;79:319-23.

7 Noishiki Y. Pattern of arrangement of smooth muscle cells in neointima of synthetic vascular prosthesis. $\mathcal{F}$ Thorac Cardiovasc Surg 1978;75:894-901.

8 Saffitz JE. Towards a unified concept of vascular smooth muscle response to injury. Am $\mathcal{f}$ Clin Pathol 1992;95: $1-2$.

9 Schwartz SM, Reidy MA. Common mechanisms of prolif eration in atherosclerosis and hypertension. Hum Patho 1987;18:240-7.

10 Malone JM, Kischer CW, Moore WS. Changes in endothelial fibrinolytic activity with venous distension and arterial implantation. $A m \mathcal{F}$ Surg 1981;142:178-82.

11 Ramos JR, Berger $\mathrm{K}$, Mansfield PB, Sauvage LR Histologic fate and endothelial changes of distended Histologic fate and endothelial changes of distended and non-28.

12 McCann RL, Larson RM, Mitchener JS, Hagen P-O Histological and biochemical studies of vascular autografts. Artery 1980;6:267-79. 